\title{
Outbreak of Anthrax Associated with Handling and Eating Meat from a Cow, Uganda, 2018
}

\author{
Esther Kisaakye, Alex Riolexus Ario, Kenneth Bainomugisha, \\ Caitlin M. Cossaboom, David Lowe, Lilian Bulage, Daniel Kadobera, Musa Sekamatte, \\ Bernard Lubwama, Dan Tumusiime, Patrick Tusiime, Robert Downing, Joshua Buule, \\ Julius Lutwama, Johanna S. Salzer, Eduard Matkovic, Jana Ritter, Joy Gary, Bao-Ping Zhu
}

On April 20, 2018, the Kween District Health Office in Kween District, Uganda reported 7 suspected cases of human anthrax. A team from the Uganda Ministry of Health and partners investigated and identified 49 cases, 3 confirmed and 46 suspected; no deaths were reported. Multiple exposures from handling the carcass of a cow that had died suddenly were significantly associated with cutaneous anthrax, whereas eating meat from that cow was associated with gastrointestinal anthrax. Eating undercooked meat was significantly associated with gastrointestinal anthrax, but boiling the meat for $>60$ minutes was protective. We recommended providing postexposure antimicrobial prophylaxis for all exposed persons, vaccinating healthy livestock in the area, educating farmers to safely dispose of animal carcasses, and avoiding handling or eating meat from livestock that died of unknown causes.

nthrax is an acute zoonotic bacterial infection Acaused by Bacillus anthracis, a gram-positive, spore-forming bacteria that is thought to survive for as long as decades in the carcasses and burial sites of infected animals (1). Anthrax is transmitted to humans through handling or eating meat from infected animal carcasses, contact with their products (e.g., hair, wool, hides, bones), or by breathing in spores $(1,2)$.

Author affiliations: Uganda Public Health Fellowship Program, Kampala, Uganda (E. Kisaakye, A. Riolexus Ario, K. Bainomugisha,

L. Bulage, D. Kadobera); Ministry of Health, Kampala

(A. Riolexus Ario, M. Sekamatte, B. Lubwama, P. Tusiime); US Centers for Disease Control and Prevention, Atlanta, Georgia, USA (C.M. Cossaboom, D. Lowe, J.S. Salzer, E. Matkovic, J. Ritter,

J. Gary, B. Zhu); Ministry of Agriculture, Entebbe, Uganda

(D. Tumusiime); Uganda Virus Research Institute, Entebbe, Uganda

(R. Downing, J. Buule, J. Lutwama); US Centers for Disease

Control and Prevention, Kampala (B.-P. Zhu)

DOI: https://doi.org/10.3201/eid2612.191373
Human anthrax infection is classified into 4 forms, depending on the route of exposure, each with a different incubation period: cutaneous (1-12 days), inhalational (1-60 days), gastrointestinal (1-6 days), and injectional (1-10 days) (3). Cutaneous anthrax is the most frequently reported form of human anthrax infection, accounting for up to $95 \%$ of cases (4). Both cutaneous and gastrointestinal anthrax outbreaks have been associated with handling or butchering infected animals and consuming their meat (5). It is estimated that each year 2,000-20,000 human anthrax cases occur worldwide (6). Most reported anthrax outbreaks occur in endemic areas in sub-Saharan Africa and Asia (1).

On April 20, 2018, the Kween District of Uganda reported to the Ministry of Health 7 suspected cases of cutaneous anthrax from 2 neighboring villages, Kaplobotwo and Rikwo. We investigated to verify the existence of an anthrax outbreak, determine its scope, identify possible exposures, and recommend evidence-based control and prevention measures.

\section{Methods}

\section{Study Area}

Kween District is located in eastern Uganda (Figure 1). It is one of the so-called "cattle-keeping corridor" districts, where cattle-rearing is a major agriculture activity.

\section{Case Definition}

For this study, we defined a suspected cutaneous anthrax case as onset of skin vesicle or eschar, $\geq 2 \mathrm{cu}-$ taneous signs and symptoms (e.g., itching, redness, swelling), or any cutaneous sign or symptom plus regional lymphadenopathy, that occurred in a resident of Kaplobotwo and Rikwo during April 11-25, 


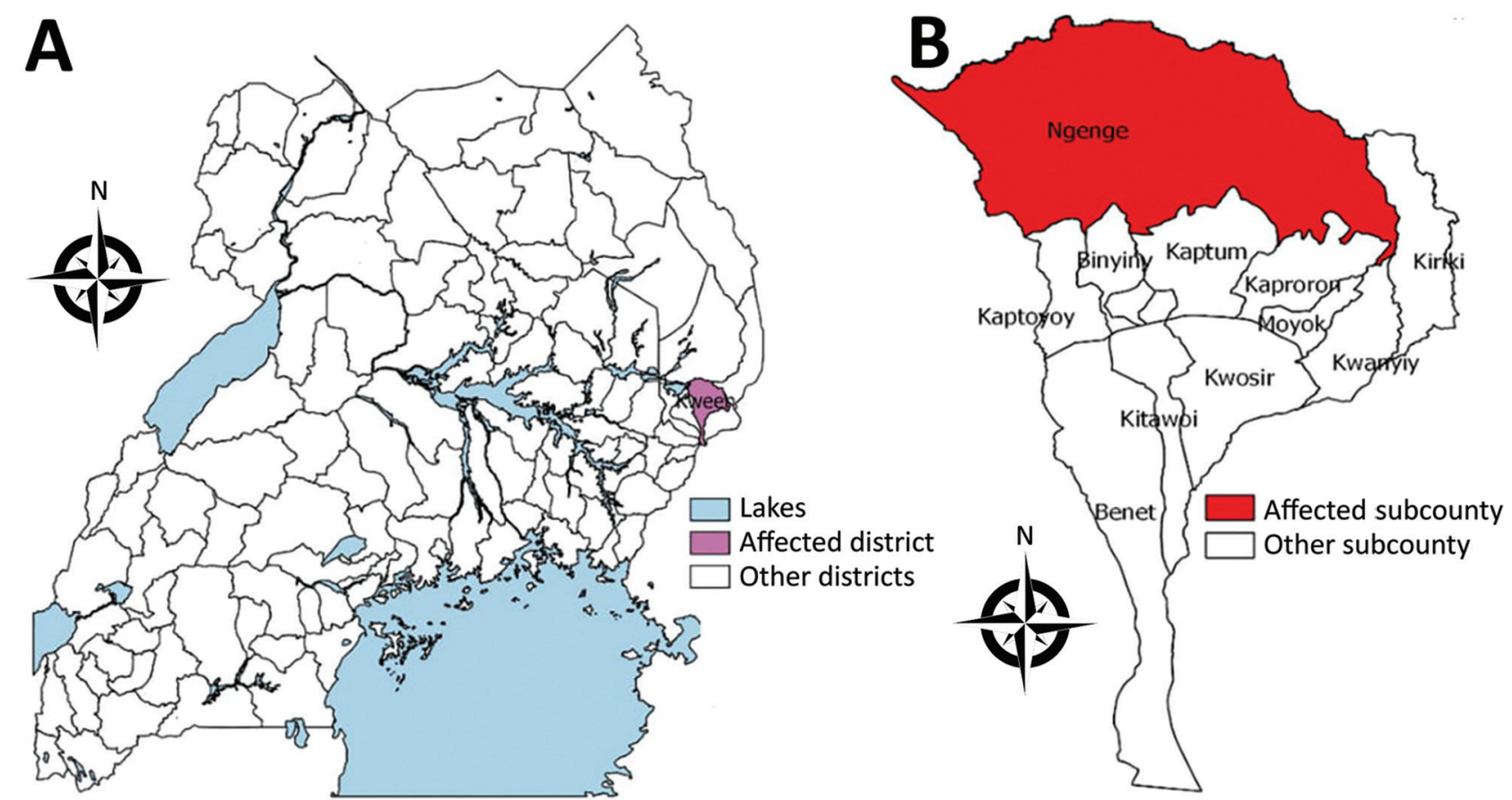

Figure 1. Area where anthrax outbreak occurred in April 2018, Kween District, Uganda. A) Location of Kween District in Uganda; B) Kween District, showing outbreak area.

2018. A suspected gastrointestinal anthrax case was defined as the acute onset of $\geq 2$ signs or symptoms: abdominal pain, vomiting, diarrhea, or sore throat. A confirmed case was a suspected case followed up with a clinical specimen (blood or swab from skin lesion or vesicle) that tested positive for $B$. anthracis by real-time PCR (rPCR).

\section{Case Identification}

To identify anthrax cases and possible fatalities, we reviewed medical records from the 3 health facilities nearest the affected communities, Ngenge Health Center III and 2 private clinics. We also conducted a house-to-house search for cases in all 57 households of the 2 affected villages with the help of village leaders and members of the village health team. We developed a list of patients with details about age, sex, residence, date of onset of any signs or symptoms, treatment provided, specimens collected, results of laboratory tests conducted, and date of discharge if the patient was hospitalized.

\section{Descriptive Epidemiology and Hypothesis Generation}

We determined the epidemiology of the outbreak by date of symptom onset, location, and demographic characteristics of patients. To identify potential exposures leading to illness, we interviewed 12 suspected anthrax case-patients using convenience sampling in Kaplobotwo. We also conducted key informant interviews with village leaders. From interviews we learned of the sudden death of a cow owned by a resident of Kaplobotwo; the cow was subsequently butchered and eaten by some villagers.

\section{Retrospective Cohort Study}

We conducted a retrospective cohort study in the moreaffected village, Kaplobotwo, where $96 \%$ of the cases occurred, focusing on exposures, which we identified using the methods described. Using a standardized questionnaire developed by the team, we interviewed villagers present in the area at the time of the outbreak. We evaluated the association between exposure to the dead cow and illness onset separately by form of anthrax illness - cutaneous, gastrointestinal, or both. We computed the attack rate (AR) and risk ratio (RR) for each activity that resulted in exposure (e.g., butchering the cow, eating the meat) to assess the association between each individual exposure and subsequent illness. Using modified Poisson regression, we also evaluated the total number of cutaneous exposures for each person interviewed relative to the risk of cutaneous anthrax to assess the dose-response relationship (7).

\section{Laboratory Investigations}

We collected 6 skin lesion swabs from patients with cutaneous-form anthrax and 8 blood specimens 
from patients with gastroenteritis-form anthrax and shipped the samples to the Uganda Virus Research Institute (UVRI; Entebbe, Uganda) for testing. The skin lesion swabs and blood specimens were tested at UVRI using rPCR following standard protocol (8).

In addition, upon revisiting the village 1 month later, we tested a specimen collected from the dried hide of the dead cow using the Active Anthrax Detect test (AAD; InBios, https://inbios.com). AAD rapid test, a novel lateral-flow rapid diagnostic test that detects the capsular polypeptide of B. anthracis, was developed as a point-of-care test for presumptive human inhalation of anthrax spores and is available as an investigational use only- or research use onlyproduct $(9,10)$. We suspended the sample in $600 \mu \mathrm{L}$ of sterile phosphate buffered saline, vortexed for 10 $\mathrm{s}$, and, after pipetting the solution multiple times, applied $10 \mu \mathrm{L}$ to the AAD cassette.

We shipped a specimen from the same dried hide to the US Centers for Disease Control and Prevention (CDC; Atlanta, GA, USA) for confirmatory testing. DNA extraction on the specimen was performed using a QIAGEN Blood Mini Kit (QIAGEN, https://www.qiagen.com), and the resulting DNA was tested using real-time reverse transcription PCR for B. anthracis from the Laboratory Reference Network (https://emergency.cdc.gov/lrn) (11). A formalin-fixed sample from the dried hide was routinely processed, embedded in paraffin, and stained with hematoxylin and eosin, Lillie-Twort gram stain, and Warthin-Starry silver stain. Immunohistochemistry assays using mouse monoclonal antibodies targeting the $B$. anthracis cell wall and capsule were performed by using an immunoalkaline phosphatase polymer system as previously described $(10,12)$.

\section{Trace-Forward Investigations and Environmental Assessment}

We conducted in-depth interviews of the district health officer, the village leader, and the owner of the dead cow, as well as a convenience sample of 15 villagers who participated in the processing of the dead cow. The interviews were conducted to investigate the circumstances surrounding the death of the cow, identify people who participated in the butchering, and determine where the meat was distributed and how many people had received the meat. We also walked through the entire village to evaluate evidence of any other dead or sick livestock in the area.

\section{Ethics Considerations}

The Office of the Director General of Health Services, Ministry of Health of Uganda, gave the directive and approval to investigate this outbreak. The Office of the Associate Director for Science, Center for Global Health, US CDC, determined that this activity was in response to a public health emergency and not human subjects research. We obtained verbal informed consent from respondents $\geq 18$ years of age or from their parents or guardians if respondents were $<18$ years of age. We stored all completed questionnaires in a secure location and stored the electronic data in a password-protected laptop to avoid disclosure of respondents' personal information. Data were not shared outside of the investigation team and when being shared within the team, all personal identifying information was deleted in advance.

\section{Results}

\section{Descriptive Epidemiology and Hypothesis Generation}

We identified 49 cases of human anthrax, 46 suspected and 3 confirmed by rPCR testing. No human deaths were reported. The mean age of the 49 patients was 30 (range 1-84) years. Of the 49 cases, 13 (27\%) had cutaneous anthrax only, $16(33 \%)$ had gastrointestinal anthrax only, and 20 (41\%) had both cutaneous and gastrointestinal anthrax. Among the 20 patients with both cutaneous and gastrointestinal anthrax, 3 had photophobia, and 2 of those 3 also had neck pain or stiffness, suggesting possible meningeal involvement (13) (Table 1).

Key informant interviews indicated that a cow had died suddenly on April 11, 2018, at the residence of a Kaplobotwo resident. Subsequently, the dead cow was skinned and butchered. Most of the adults in Kaplobotwo participated in the butchering and handling of the meat, and many villagers took the meat home to eat. Some of the meat was also sold to neighboring Rikwo. According to local leaders, Kaplobotwo had a total of 234 residents and Rikwo a total of 120 residents. When we analyzed the geographic locations of the cases, 47 (96\%) occurred in Kaplobotwo (AR 20\%, 47/234) and 2 (4.1\%) occurred in Rikwo (AR 1.7\%, 2/120). In Kaplobotwo, all cases were within a 600-meter radius of the site where the dead cow was skinned and butchered.

The epidemic curve showed that, after the death and processing of the cow, cases began to appear on April 13, and the number of cases rose, peaking on April 15, suggesting a point-source outbreak. After that, the onset of cases declined, the last being on April 25 (Figure 2, panel A). When the epidemic curve was stratified by anthrax forms (Figure 2, panels B-D), the intervals from exposure to the peak of the epidemic curve was 3 days for cutaneous and 2 days for 


\begin{tabular}{|c|c|c|c|c|}
\hline \multirow[b]{2}{*}{ Signs and symptoms } & \multicolumn{4}{|c|}{ No. (\%) patients } \\
\hline & All cases, $\mathrm{N}=49$ & $\begin{array}{c}\text { Cutaneous-only, } \\
n=13\end{array}$ & $\begin{array}{c}\text { Gastrointestinal-only, } \\
n=16\end{array}$ & Both, $n=20$ \\
\hline \multicolumn{5}{|l|}{ Cutaneous } \\
\hline Skin itching (pruritis) & $35(65)$ & $12(92)$ & 0 & $20(100)$ \\
\hline Skin reddening (erythema) & $25(51)$ & $12(92)$ & 0 & $13(65)$ \\
\hline Skin swelling (edema) & $26(53)$ & $11(85)$ & 0 & $15(75)$ \\
\hline Skin vesicles & $17(35)$ & $8(62)$ & 0 & $9(45)$ \\
\hline Skin eschar & $9(18)$ & $3(23)$ & 0 & $6(30)$ \\
\hline Regional lymphadenopathy & $15(31)$ & $4(31)$ & 0 & $11(55)$ \\
\hline \multicolumn{5}{|l|}{ Gastrointestinal } \\
\hline Abdominal pain & $37(76)$ & $2(15)$ & $16(100)$ & $19(95)$ \\
\hline Diarrhea & $28(57)$ & 0 & $12(75)$ & $16(80)$ \\
\hline Bloody diarrhea & $9(18)$ & 0 & $6(38)$ & $3(15)$ \\
\hline Sore throat & $13(27)$ & 0 & $6(38)$ & $7(35)$ \\
\hline Vomiting & $10(20)$ & 0 & $3(19)$ & $7(35)$ \\
\hline Swollen neck lymph nodes & $2(4.1)$ & 0 & $1(6.3)$ & $1(5.0)$ \\
\hline \multicolumn{5}{|l|}{ Systemic } \\
\hline Fever & $25(51)$ & $2(15)$ & $8(50)$ & $15(75)$ \\
\hline Lethargy & $24(49)$ & $1(7.7)$ & $9(56)$ & $14(70)$ \\
\hline Anorexia & $13(27)$ & 0 & $6(38)$ & $7(35)$ \\
\hline Difficulty breathing & $5(10)$ & 0 & $1(6.3)$ & $4(20)$ \\
\hline Cough & $5(10)$ & 0 & $1(6.3)$ & $4(20)$ \\
\hline Headache & $3(6.1)$ & 0 & 0 & $3(15)$ \\
\hline \multicolumn{5}{|l|}{ Other } \\
\hline Photophobia & $3(6.1)$ & 0 & 0 & $3(15)$ \\
\hline Neck pain or stiffness & $2(4.1)$ & 0 & 0 & $2(10)$ \\
\hline
\end{tabular}

gastrointestinal. The interval from the initial exposure (April 11-12, 2018) to the end of the epidemic curve was 12-13 days for onset of cutaneous anthrax and 8-9 days for onset of gastrointestinal anthrax. Of the 12 suspected case-patients who participated in the hypothesis-generation interview, 100\% carried and ate the meat of the dead cow and were involved in the cutting or butchering, $50 \%$ participated in the cleaning of the waste site after the carcass was processed, and $33 \%$ participated in skinning the dead cow before butchering.

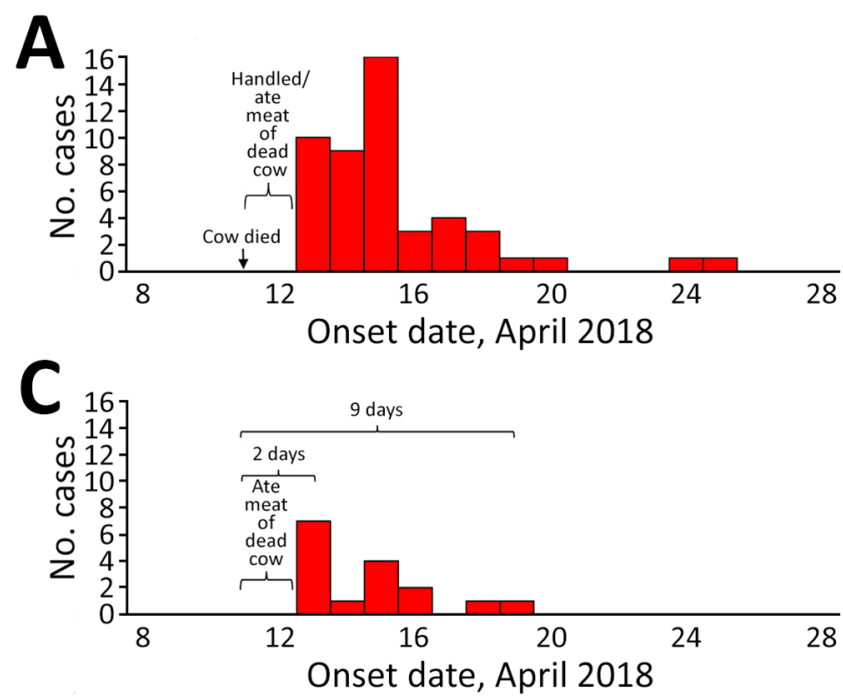

\section{Retrospective Cohort Study Findings}

In our retrospective cohort study in Kaplobotwo, we interviewed 141 persons who resided in the village during April 2018 and therefore could have been exposed to anthrax. Among these 141 villagers, anthrax developed in 47 (AR 33\%); cutaneous anthrax developed in 33 (AR 23\%), and gastrointestinal anthrax developed in 34 (AR 24\%). By anthrax form, the ARs were $9.2 \%$ for cutaneous-only, $9.9 \%$ for gastrointestinal-only, and $14 \%$ for combined cutaneous and gastrointestinal anthrax.
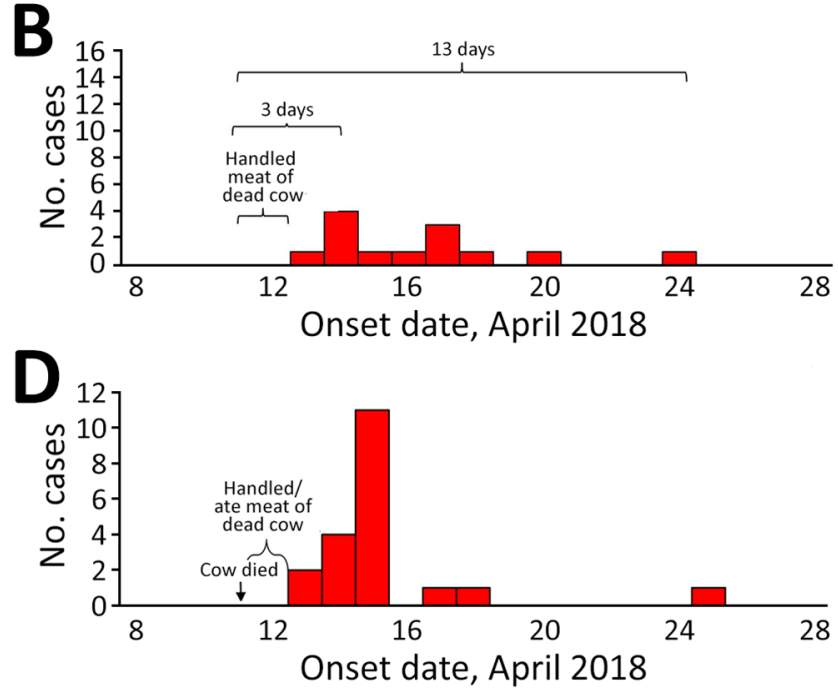

Figure 2. Distribution by date of onset of cases in anthrax outbreak that occurred in April 2018, Kween District, Uganda. A) All anthrax cases; B) cutaneous-only cases; C) gastrointestinal-only cases; D) cases of both cutaneous and gastrointestinal anthrax. 
Male residents had a lower AR than female residents for cutaneous-only anthrax $(6.5 \%$ vs. $13 \%)$; however, this difference was not significant $(p=0.175$ by Fisher exact test). Conversely, male residents had higher ARs than female residents for both the gastrointestinal-only form ( $16 \%$ vs. $3.1 \%$; $p=0.012$ by Fisher exact test) and the combined cutaneous and gastrointestinal form $(19 \%$ vs. $7.8 \%$; $p=0.039$ by Fisher exact test). The cutaneous-only form affected adults more than children, whereas the gastrointestinal-only form affected young children ( $\leq 5$ years) and older adults ( $\geq 30$ years) more than older children (5-17 years) and young adults (18-29 years). The mixed cutaneous and gastrointestinal form affected all age groups approximately equally (Table 2 ).

In the retrospective cohort study, certain activities were significantly associated with developing cutaneous anthrax: carrying the dead cow from the place of death to the place of butchering (RR 4.3, 95\% CI 2.4-7.8), participating in the skinning of the cow (RR 4.2, 95\% CI 2.6-6.7), participating in cutting and butchering of the dead cow (RR 4.9, 95\% CI 3.2-7.9), participating in the removal of the organs (RR 3.5, 95\% CI 2.1-6.0), carrying the skin of the dead cow from the butchering site to homes (RR 4.5, 95\% CI 2.9-6.9), carrying the cut meat from the place of butchering to homes (RR 4.3, 95\% CI 2.4-7.8), and cleaning the waste site after the butchering (RR 4.2, 95\% CI 2.6-6.7). The number of cutaneous exposures for each person ranged from 0 to 7 . Of the 141 persons who participated in the cohort study, 99 (70\%) had no exposures at all, $6(4.3 \%)$ reported only 1 exposure, $22(16 \%)$ reported 2 exposures, 4 (2.8\%) reported 3 exposures, 2 (1.4\%) reported 4 exposures, 1 (0.71\%) reported 5 exposures, $2(1.4 \%)$ reported 6 exposures, and $5(3.6 \%)$ reported having all 7 exposures. For each additional cutaneous exposure, the risk for cutaneous anthrax increased by 30\% (RR 1.4, 95\% CI 1.3-1.5).

Eating meat from the dead cow was significantly associated with gastrointestinal anthrax (RR $¥, 95 \% \mathrm{CI}$ $4.3 ¥$ by Fisher exact test; $p=0.00$ ). Of the 95 persons who ate the cow meat, eating meat that was boiled for $\leq 30$ minutes was significantly associated with gastrointestinal anthrax (RR 2.5, 95\% CI 1.5-4.1); we found that boiling meat for $>60$ minutes was protective compared with the shorter cooking time (RR $0.34,95 \%$ CI 0.18-0.67) (Table 3).

\section{Laboratory Investigation Findings}

Of the 6 skin lesion swabs collected, 3 tested positive for $B$. anthracis DNA by rPCR at UVRI. All 8 blood samples were negative for $B$. anthracis by rPCR at UVRI. It should be noted that, at the time of specimen
Table 2. Anthrax attack rates by age and sex for each form, Kaplobotwo, Kween District, Uganda, April 2018*

\begin{tabular}{|c|c|c|c|}
\hline Anthrax form & $\begin{array}{l}\text { Total } \\
\text { cohort }\end{array}$ & $\begin{array}{c}\text { No. } \\
\text { cases }\end{array}$ & AR, $\%$ \\
\hline All anthrax & 141 & 47 & 33 \\
\hline \multicolumn{4}{|l|}{ Sex } \\
\hline M & 77 & 32 & 42 \\
\hline $\mathrm{F}$ & 64 & 15 & 23 \\
\hline \multicolumn{4}{|l|}{ Age range, y } \\
\hline $0-5$ & 30 & 12 & 40 \\
\hline $6-17$ & 27 & 6 & 22 \\
\hline $18-29$ & 27 & 7 & 26 \\
\hline $30-59$ & 40 & 16 & 40 \\
\hline$\geq 60$ & 17 & 6 & 35 \\
\hline Cutaneous-only & 141 & 13 & 9.2 \\
\hline \multicolumn{4}{|l|}{ Sex } \\
\hline$M$ & 77 & 5 & 6.5 \\
\hline $\mathrm{F}$ & 64 & 8 & 13 \\
\hline \multicolumn{4}{|l|}{ Age range, $y$} \\
\hline $0-5$ & 30 & 1 & 3.3 \\
\hline $6-17$ & 27 & 1 & 3.7 \\
\hline $18-29$ & 27 & 3 & 11 \\
\hline $30-59$ & 40 & 6 & 15 \\
\hline$\geq 60$ & 17 & 2 & 12 \\
\hline Gastrointestinal-only & 141 & 14 & 9.9 \\
\hline \multicolumn{4}{|l|}{ Sex } \\
\hline$M$ & 77 & 12 & 16 \\
\hline $\mathrm{F}$ & 64 & 2 & 3.1 \\
\hline \multicolumn{4}{|l|}{ Age range, y } \\
\hline $0-5$ & 30 & 5 & 17 \\
\hline $6-17$ & 27 & 1 & 3.7 \\
\hline $18-29$ & 27 & 1 & 3.7 \\
\hline $30-59$ & 40 & 5 & 13 \\
\hline$\geq 60$ & 17 & 2 & 12 \\
\hline Cutaneous and gastrointestinal & 141 & 20 & 14 \\
\hline \multicolumn{4}{|l|}{ Sex } \\
\hline M & 77 & 15 & 19 \\
\hline $\mathrm{F}$ & 64 & 5 & 7.8 \\
\hline \multicolumn{4}{|l|}{ Age range, $y$} \\
\hline $0-5$ & 30 & 6 & 20 \\
\hline $6-17$ & 27 & 4 & 15 \\
\hline $18-29$ & 27 & 3 & 11 \\
\hline $30-59$ & 40 & 5 & 13 \\
\hline$\geq 60$ & 17 & 2 & 12 \\
\hline
\end{tabular}

collection, all patients had already started and some had completed antimicrobial treatment. A sample from the dried hide of the cow, taken 1 month after the initial visit to the village, tested positive by AAD in the field and was confirmed to be positive for $B$. anthracis by both $\mathrm{rPCR}$ and immunohistochemistry at CDC.

\section{Trace-Forward and Environmental Investigation}

According to the village leader, after the cow died on April 11, 2018, a total of 10 residents of Kaplobotwo participated in butchering, skinning, and carrying meat from the cow, and most of the villagers ate meat from the dead cow. Environmental investigations found that the village was near the Panupe Game Reserve. Piles of animal bones were found in the livestock grazing fields, indicating past animal 
deaths. Interviews with community leaders revealed that these were remains from animals that had died suddenly and were abandoned in the grazing fields.

Some of the meat from the dead cow was reportedly sold to 2 neighboring villages, Rikwo and Tukumo. Due to resource limitations, we were unable to conduct house-to-house searches for cases in these 2 villages; instead, we contacted the village leaders for case finding. In Rikwo, a family of 2 bought the meat from a meat broker, and gastrointestinal symptoms developed in both family members after they ate the meat. The owner of a bar in the same village also bought the meat, boiled it overnight, poured out the broth from the boiling pot the next morning, fried the boiled meat, and sold it to 28 patrons the next day. None of the patrons reported any gastrointestinal symptoms. In Tukumo, the meat was sold to a bar, a restaurant, and an unknown number of individual families. The village leader was aware of 23 persons who bought and ate the meat; however, he did not know of anyone who had reported gastrointestinal or cutaneous anthrax symptoms.

\section{Discussion}

On the basis of epidemiologic, laboratory, and environmental assessments, we determined that this was a point-source cutaneous and gastrointestinal human anthrax outbreak associated with handling and eating meat from a cow that had died from confirmed anthrax infection. Results from this investigation were consistent with those in other anthrax outbreak investigations in which anthrax patients were infected through contact with diseased livestock or contaminated animal products (14-17).

In our study, although the cause of the cow's death was unknown at the time of death, subsequent laboratory testing confirmed anthrax in the dried hide of the cow. In this area, when a cow is butchered, it is customary to share meat with all households in the village. In this case, this custom exposed the entire village to anthrax. Butchering anthrax-infected animals and disposing of carcasses and waste in environments where ruminants live and graze, combined with limited vaccination of livestock against anthrax, enables further environmental contamination with $B$. anthracis spores and propagation of anthrax outbreaks in animals and zoonotic transmission to humans (18). Findings from this investigation are consistent with findings from a previous study in Kuwirirana ward, Gokwe North, Zimbabwe, in which anthrax also resulted from contact with and consumption of anthrax-infected carcasses (19).

Among people, anthrax infection is typically an occupational disease, most common among farmers and workers with occupational activities that involve handling animals and animal products, such as the herders, butchers, and others. Infections may also occur among persons who consume infected meat $(4,20,21)$. In this outbreak, cutaneous-only anthrax affected adults more than children, probably because adults were more likely to have been engaged in handling and processing the dead cow.

Spores of B. anthracis are refractory to inactivation by boiling and, in this outbreak, eating undercooked meat was significantly associated with developing gastrointestinal anthrax. Conversely, boiling meat for $>60$ minutes appeared to be protective among persons who ate it, possibly because that length of time could have allowed the heat to rise to a temperature sufficient to inactivate a portion of the spores. Whether or not this actually occurred is unclear. Findings in this study are consistent with those found in a study in Bangladesh in which high rates of cutaneous anthrax but few gastrointestinal anthrax cases occurred in a community that had cooked the meat longer (22).

In addition, the risk for gastrointestinal anthrax remained high even when the meat was well cooked (AR $31 \%$ ) or boiled for $>60$ minutes (AR 22\%). According to World Health Organization guidelines, "any animal that is sick, behaves strangely or has died suddenly should not be used for food or for making any product, as it may have succumbed to an

\begin{tabular}{|c|c|c|c|c|c|}
\hline \multirow[b]{2}{*}{ Form } & \multicolumn{2}{|c|}{ Cases } & \multicolumn{2}{|c|}{ Attack rate, $\%$} & \multirow[b]{2}{*}{$\operatorname{RR}(95 \% \mathrm{Cl})$} \\
\hline & Exposed & Nonexposed & Exposed & Nonexposed & \\
\hline \multicolumn{6}{|l|}{ Cutaneous anthrax } \\
\hline Carried dead cow & 37 & 104 & 54 & 13 & $4.3(2.4-7.8)^{*}$ \\
\hline Participated in skinning & 10 & 131 & 80 & 19 & $4.2(2.6-6.7)^{*}$ \\
\hline Participated in cutting/butchering & 10 & 131 & 90 & 18 & $4.9(3.2-7.5)^{*}$ \\
\hline Participated in removing organs & 10 & 131 & 70 & 20 & $3.5(2.1-6.0)^{*}$ \\
\hline Carried the skin of the dead cow & 8 & 133 & 88 & 20 & $4.5(2.9-6.9)^{*}$ \\
\hline Carried cut meat & 37 & 104 & 54 & 13 & $4.3(2.4-7.8)^{*}$ \\
\hline Cleaned the waste & 10 & 131 & 80 & 19 & $4.2(2.6-6.7)^{*}$ \\
\hline For every additional exposure* & & & & & $1.4(1.3-1.5)^{*}$ \\
\hline \multicolumn{6}{|l|}{ Gastrointestinal anthrax } \\
\hline Ate meat from dead cow, total & 95 & 46 & 35 & 0 & $\infty(4.3-\infty)^{*}$ \\
\hline
\end{tabular}


infectious disease" (23). Following these guidelines can safeguard both animal products and persons involved in handling them.

This study had some limitations. In this outbreak, B. anthracis was confirmed by rPCR in 3 of the 6 skinlesion swab specimens, as well as from the dried hide of the cow. However, the 8 blood specimens from patients with gastroenteritis were negative for B. anthracis by both rPCR and culture. These negative findings might be explained by the fact that all patients were already under antimicrobial treatment at the time of specimen collection. Whereas clinical and epidemiologic characteristics strongly suggested gastrointestinal anthrax, we were unable to provide definitive proof without laboratory confirmation. Clinical signs and symptoms of both cutaneous and gastrointestinal anthrax are nonspecific; therefore, some of the identified cases found might actually have been noncases. In addition, the dried hide of the implicated cow tested positive by AAD rapid test. There is great utility for a rapid diagnostic test for presumptive diagnosis of anthrax under field conditions, but care must be taken when interpreting the results of this test. Recent work has identified that the specificity of this assay decreases with carcass age ( $>24$ hours after death), so parallel confirmatory testing is critical when interpreting results from this test (24). Also, trace-forward investigation indicated that some meat from the implicated cow might have been sold to neighboring villages, but no house-to-house search was conducted in those villages, possibly resulting in undercounting of cases.

This investigation highlights an outbreak of human cutaneous and gastrointestinal anthrax among persons handling and eating meat from a cow that died of presumed anthrax. As a result of our findings, we made several recommendations to the communities: routinely vaccinate livestock; continue education and mobilization for anthrax; administer antimicrobials to all persons identified with anthrax and prophylaxis to exposed community members; use rapid diagnostic tests at the district level to quickly provide presumptive evidence of anthrax in animal carcasses; and safely bury carcasses under supervision. For burial, carcasses should be disinfected at the site of death with $12.5 \%$ formalin solution and buried in a pit $\geq 6$ feet deep with the bottom of the pit $\geq 3$ feet above the water table. We also recommended building capacity and the awareness of healthcare workers to obtain samples from patients before beginning drug administration.

The investigation team worked with the district to conduct community health education on these rec- ommendations and about the dangers of eating meat from animals found dead. We also provided antimicrobial treatment (ciprofloxacin and doxycycline) to all identified patients, offered postexposure antimicrobial prophylaxis to carcass-disposal team members and exposed community members, replenished antimicrobials at Ngenge Health Center III, and provided personal protective equipment and training in its use to the carcass disposal teams. Finally, we advocated for prompt reporting of suspected anthrax cases to the district health office, district veterinary office, and the national One Health coordinator.

\section{Acknowledgments}

We are grateful to the Kween District Rapid Response Team for their contribution to the outbreak investigation and response. We thank the staff at the Public Health Emergency Operation Centre and National Task Force in Epidemic Preparedness and Response, Ministry of Health, for coordinating the outbreak response, and staff of Makerere University School of Public Health for their technical support. We also thank all who managed the specimen transport system for ensuring the smooth operations of the system, and staff of CDC's National Center for Emerging and Zoonotic Infectious Diseases, Office of the Deputy Director for Infectious Diseases, Bacterial Special Pathogens Branch (specifically Antonio R. Vieira, William A. Bower, Cari B. Kolton, Chung K. Marston, Robyn A. Stoddard, and Alex R. Hoffmaster) for providing technical assistance and support with the laboratory investigations. Finally, we acknowledge Syamal Raychaudhuri at InBios for providing the AAD lateral flow cassettes.

\section{About the Author}

Esther Kisaakye is a field epidemiology training program fellow in the 2018 cohort of the Uganda Public Health Fellowship program. She is currently hosted at the Uganda Ministry of Health to monitor, investigate, and control disease outbreaks in the country, and to conduct disease and mortality surveillance activities.

\section{References}

1. Turnbull PCB. Introduction: anthrax history, disease and ecology. In: Koehler TM, editor. Anthrax. Current topics in microbiology and immunology, vol 271. Berlin: Springer; 2002. p. 1-19.

2. Dragon DC, Rennie RP. The ecology of anthrax spores: tough but not invincible. Can Vet J. 1995;36:295-301.

3. Chin J, editor. Control of communicable diseases manual. 17th ed. Washington: American Public Health Association; 2000.

4. Hicks CW, Sweeney DA, Cui X, Li Y, Eichacker PQ. An overview of anthrax infection including the recently 
identified form of disease in injection drug users. Intensive Care Med. 2012;38:1092-104. https://doi.org/10.1007/ s00134-012-2541-0

5. Inglesby TV, O'Toole T, Henderson DA, Bartlett JG, Ascher MS, Eitzen E, et al.; Working Group on Civilian Biodefense. Anthrax as a biological weapon, 2002: updated recommendations for management. JAMA. 2002;287:2236-52. https:/ / doi.org/10.1001/jama.287.17.2236

6. Martin GJ, Friedlander AM. Bacillus anthracis (anthrax). In: Mandell GL, Bennett JE, Dolin R, editors. Mandell, Douglas, and Bennett's principles and practice of infectious diseases. 7th ed. Philadelphia: Elsevier; 2010. p. 2715-25.

7. Yelland LN, Salter AB, Ryan P. Performance of the modified Poisson regression approach for estimating relative risks from clustered prospective data. Am J Epidemiol. 2011;174:984-92. https://doi.org/10.1093/aje/kwr183

8. Oggioni MR, Meacci F, Carattoli A, Ciervo A, Orru G, Cassone A, et al. Protocol for real-time PCR identification of anthrax spores from nasal swabs after broth enrichment. J Clin Microbiol. 2002;40:3956-63. https:/ / doi.org/10.1128/ JCM.40.11.3956-3963.2002

9. Gates-Hollingsworth MA, Perry MR, Chen H, Needham J, Houghton RL, Raychaudhuri S, et al. Immunoassay for capsular antigen of Bacillus anthracis enables rapid diagnosis in a rabbit model of inhalational anthrax. PLoS One. 2015;10:e0126304. https://doi.org/10.1371/journal. pone. 0126304

10. Guarner J, Jernigan JA, Shieh W-J, Tatti K, Flannagan LM, Stephens DS, et al.; Inhalational Anthrax Pathology Working Group. Pathology and pathogenesis of bioterrorism-related inhalational anthrax. Am J Pathol. 2003;163:701-9. https://doi.org/10.1016/S0002-9440(10)63697-8

11. Hoffmaster AR, Meyer RF, Bowen MD, Marston CK, Weyant RS, Thurman K, et al. Evaluation and validation of a real-time polymerase chain reaction assay for rapid identification of Bacillus anthracis. [Erratum in: Emerg Infect Dis. 2003;9:511.] Emerg Infect Dis. 2002;8:1178-82. https://doi.org/10.3201/eid0810.020393

12. Bollweg BC, Silva-Flannery L, Spivey P, Hale GL. Optimization of commercially available Zika virus antibodies for use in a laboratory-developed immunohistochemical assay. J Pathol Clin Res. 2017;4:19-25. https:// doi.org/10.1002/cjp2.84

13. Levy LM, Baker N, Meyer MP, Crosland P, Hampton J. Anthrax meningitis in Zimbabwe. Cent Afr J Med. 1981;27:101-4.

14. Mwakapeje ER, Høgset S, Softic A, Mghamba J, Nonga HE, Mdegela RH, et al. Risk factors for human cutaneous anthrax outbreaks in the hotspot districts of Northern Tanzania: an unmatched case-control study. R Soc Open Sci. 2018;5:180479. https:/ / doi.org/10.1098/ rsos.180479

15. Yu D, He J, Zhang E, Wang P, Liu D, Hou Y, et al. Investigation and source-tracing of an anthrax outbreak in Gansu Province, China. PLoS One. 2018;13:e0203267. https:/ / doi.org/10.1371/journal.pone.0203267

16. Mwenye KS, Siziya S, Peterson D. Factors associated with human anthrax outbreak in the Chikupo and Ngandu villages of Murewa district in Mashonaland East Province, Zimbabwe. Cent Afr J Med. 1996;42:312-5.

17. Islam MS, Hossain MJ, Mikolon A, Parveen S, Khan MSU, Haider N, et al. Risk practices for animal and human anthrax in Bangladesh: an exploratory study. Infect Ecol Epidemiol. 2013;3:21356. https://doi.org/10.3402/iee.v3i0.21356

18. Rume FI. Epidemiology of anthrax in domestic animals of Bangladesh [PhD thesis]. Dhaka, Bangladesh: University of Dhaka; 2018.

19. Gombe NT, Nkomo BMM, Chadambuka A, Shambira G, Tshimanga M. Risk factors for contracting anthrax in Kuwirirana ward, Gokwe North, Zimbabwe. Afr Health Sci. 2010;10:159-64.

20. Jong EC, Stevens DL, editors. Netteris infectious disease, 1st ed. Philadelphia: Elsevier-Saunders, 2011.

21. Smyth HF, Cheney VS. Anthrax as an occupational disease. Am J Public Health Nations Health. 1930;20:155-60. https:/ / doi.org/10.2105/ AJPH.20.2.155

22. Chakraborty A, Khan SU, Hasnat MA, Parveen S, Islam MS, Mikolon A, et al. Anthrax outbreaks in Bangladesh, 20092010. Am J Trop Med Hyg. 2012;86:703-10. https:/ / doi.org/ 10.4269/ajtmh.2012.11-0234

23. World Health Organization. Anthrax: questions and answers. 2019 [cited 2019 July 19]. http:/ / www.euro.who. int/en/health-topics/disease-prevention/food-safety/ data-and-statistics/anthrax-questions-and-answers

24. Kolton CB, Marston CK, Stoddard RA, Cossaboom C, Salzer JS, Kozel TR, et al. Detection of Bacillus anthracis in animal tissues using InBios active anthrax detect rapid test lateral flow immunoassay. Lett Appl Microbiol. 2019;68:4804. https://doi.org/10.1111/lam.13134

Address for correspondence: Esther Kisaakye, Uganda Public Health Fellowship Program, Ministry of Health, 4th Floor, Lourdel Towers, Plot 1, Lourdel Rd, Nakasero, P.O. Box 7072, Kampala, Uganda; email: estherkisaakye@musph.ac.ug 